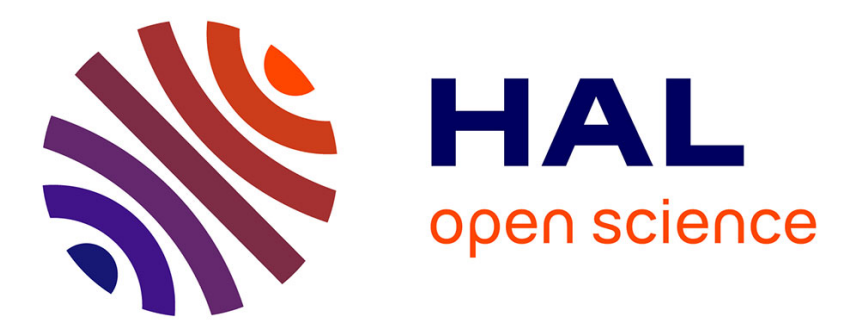

\title{
Les agricultures paysannes extrapampéennes argentines. Au-delà des résistances
}

\author{
Christophe Albaladejo, Roberto Cittadini, Pedro Carricart, Patricio Sanz, \\ Roberto Bustos Cara, Hernán Hernández, Amalia Sapag
}

\section{To cite this version:}

Christophe Albaladejo, Roberto Cittadini, Pedro Carricart, Patricio Sanz, Roberto Bustos Cara, et al. Les agricultures paysannes extrapampéennes argentines. Au-delà des résistances. L'agriculture en famille: travailler, ré-inventer, transmettre, EDP Sciences, 2014. hal-01595577

\section{HAL Id: hal-01595577 \\ https://hal.science/hal-01595577}

Submitted on 26 Sep 2017

HAL is a multi-disciplinary open access archive for the deposit and dissemination of scientific research documents, whether they are published or not. The documents may come from teaching and research institutions in France or abroad, or from public or private research centers.
L'archive ouverte pluridisciplinaire HAL, est destinée au dépôt et à la diffusion de documents scientifiques de niveau recherche, publiés ou non, émanant des établissements d'enseignement et de recherche français ou étrangers, des laboratoires publics ou privés.

\section{다(1)(2)}

Distributed under a Creative Commons Attribution - ShareAlikel 4.0 International 


\title{
Les agricultures paysannes extrapampéennes argentines. Au-delà des résistances
}

\author{
Roberto Cittadini ${ }^{1}$, Pedro Carricart ${ }^{2}$, \\ Roberto Bustos Cara ${ }^{3}$, Hernán Hernández ${ }^{4}$, \\ Amalia Sapag $^{5}$, Patricio Sanz ${ }^{6}$, Christophe Albaladejo ${ }^{7}$
}

\section{Introduction $^{8}$}

En Argentine, la modernisation agricole des années 1960 s'est essentiellement concentrée sur l'exploitation familiale moyenne capitalisée. Durant plus de 30 ans, la pensée dominante a considéré que les exploitants devaient se transformer en "producteurs agricoles modernes ", autrement dit en un personnage idéal-typique devenu la référence de la modernité et de la professionnalisation de l'agriculture. Cependant, ce modèle n'a pas réussi à s'imposer en dehors de la région pampéenne. En effet, les régions extra-pampéennes (à l'exception des périmètres irrigués) sont classiquement présentées comme des réservoirs d'agricultures dites " traditionnelles " (paysannes ou latifundiaires), voire archaïques, en tout cas réfractaires au développement. Les petits exploitants de ces régions ont le plus souvent été assimilés à des reliques, ou au mieux à des formes de résilience, voire des résistances. C'est ce qui transparait des projets de développement rural développés, à l'époque, par l'Institut national de la technologie agricole (INTA), les ministères de l'Agriculture des provinces, les ONG ou encore le Conseil fédéral des investissements (CFI). Cette vision en termes de tradition et/ou de résistance a aussi indirectement prévalu dans la grande majorité

1. INTA Labintex Europa, UMR 951 Innovation, Montpellier ; cittadini.roberto@inta.gob.ar

2. UNLP Facultad de Ciencias Agrarias y Forestales, y Asociación de Cooperativas Argentinas (Argentine) ; pedrocarricart@gmail.com

3. UNS Departamento de Geografía y Turismo (Argentine) ; usbustos@gmail.com

4. Proyecto Regional con Enfoque Territorial PReT Chaco Norte. INTA - Agencia de Extensión Rural Tartagal, Salta (Argentine); hernandez.hernan@inta.gob.ar

5. Dirección Provincial de Desarrollo Rural, Provincia de Neuquen (Argentine); amaliasapag@yahoo.com.ar

6. INTA EEA Ing. Guillermo N. Juárez ; sanz.patricio@inta.gob.ar

7. INRA, Département de recherche Sciences pour l'action et le développement (France) et UNLP Facultad de Ciencias Agrarias y Forestales (Argentine) ; christophealbaladejo@icloud.com 8. Ce travail a bénéficié d'une aide de l'Agence nationale de la recherche et de l'Agence interinstituts de recherche pour le développement dans le cadre du programme SYSTERRA, portant la référence ANR-09-STRA-04. Tous les auteurs font partie du laboratoire international Agriterris.

Ce chapitre de l'ouvrage L'agriculture en famille : travailler, réinventer, transmettre est publié en Open Access sous licence creative commons CC-BY-NC-ND permettant l'utilisation non commerciale, la distribution, la reproduction 
des études et des débats académiques qui ont mis en évidence des agricultures paysannes en tant que formes sociales, économiques et techniques cohérentes, mais qui les ont interprétées en grande partie comme des émanations de la tradition et/ou de la pauvreté (Basco, et al., 1981 ; Bendini De Ortega, et al., 1985 ; Giarracca, 1990 ; Murmis, 1993 ; Jong et Tiscornia, 1994 ; Domínguez, 2005).

Pourtant, depuis l'émergence, au début des années 2000 en Argentine, d'un mouvement politique et social "d'agriculture familiale " ${ }^{9}$, ces petites agricultures extrapampéennes ne cessent de rendre visibles des discours, des formes associatives, des modes d'exploitation des ressources naturelles, des relations au développement qui ne peuvent être assimilées à des archaïsmes ou des survivances du passé, ni non plus à des résistances face à un modèle dominant, et qui apparaissent bien au contraire comme de nouveaux modes d'articulation au territoire.

Ce travail de recherche, basé sur une recherche-action avec des agents de développement agricole, montre l'originalité et l'inventivité de ces agricultures qui le plus souvent s'autodésignent "paysannes ", et l'importance de dépasser une vision en termes de " résistances " ou de "tradition " pour analyser les nouveaux modes de production, de vie et d'action collective contribuant à une construction originale, voire totalement inédite, du territoire et de l'activité en émergence.

La recherche prend appui sur la direction de travaux d'une vingtaine d'étudiants, tous intégrés dans une même équipe de recherche (le laboratoire Agriterris), dans trois régions argentines de fortes « agricultures paysannes " : la Patagonie, le NordOuest et le Nord-Est. Tous les étudiants sont des agents de développement réalisant une thèse de master comportant l'équivalent d'un travail de 18 mois de recherche à temps plein. Nous analysons ici trois cas correspondant à trois mémoires d'étudiants, co-auteurs de ce chapitre ${ }^{10}$, afin d'illustrer nos propos.

L’analyse des travaux de recherche développés par ces agents de développement concernant les agricultures familiales comporte trois grandes dimensions :

- l'action collective, et en particulier l'action associative, les formes de participation à la gouvernance du développement et du territoire ;

- la gestion des ressources naturelles : pratiques individuelles et collectives et plus globalement les savoirs locaux concernant la nature et sa gestion ;

- les relations au monde du développement, à l'État et aux sources de financement, de technologie et d'information.

9. En 2004, et en suivant l'exemple de leur voisin brésilien, plus de 900 organisations rurales du pays réussissent à organiser un espace de débat et de concertation dénommé Forum national de l'agriculture familiale (FoNAF) à partir duquel se construit et s'institutionnalise une nouvelle catégorie politique de l'" Agriculture familiale ». En 2009, le gouvernement argentin crée le Secrétariat de l'agriculture familiale et du développement rural, consacrant ainsi la reconnaissance officielle d'un nouvel acteur du secteur agricole et des transformations des politiques publiques. C'est un événement marquant de l'histoire agraire de l'Argentine de grande portée symbolique puisqu'il implique un reversement de la domination absolue de l'idéologie de la modernisation durant les dernières décennies (Gisclard et Allaire, 2012).

10. Hernán Hernández, Amalia Sapag et Patricio Sanz. 


\section{Modernisation et modernités des agricultures familiales argentines}

Nous mobiliserons pour notre analyse le cadre conceptuel de la géographie sociale française, et notamment la théorie des médiations territoriales développée par Albaladejo (2004, 2009), dont nous rappelons ici deux aspects susceptibles de nous aider à interpréter les transformations en cours de l'agriculture familiale :

- l'activité agricole est comprise au sens large d'activité humaine et n'est donc pas considérée uniquement comme une activité de production physique et économique : elle est une activité de production de la personne (des identités individuelles et des projets des individus), de production de la vie politique (discours, représentations, associations, etc.) et de reproduction de la vie matérielle (de biens matériels mais aussi d'organisation et d'identités au travail). Elle est donc une activité de production de la société et du territoire. C'est à travers ces trois dimensions, qu'Albaladejo propose de mettre en parallèle avec les concepts d'œuvre, d'action et de travail d'Hannah Arendt (1994), que nous analyserons les transformations des agricultures paysannes ;

- les innovations en agriculture ne sont pas uniquement techniques ou productives, elles sont aussi sociales, culturelles et politiques. Bien que ces différentes dimensions du changement soient toujours en étroite relation, elles ne vont pas toujours de pair. C'est ainsi qu'Albaladejo (2009) propose d'analyser les décalages entre les processus de " modernisation " (sous-entendu technique et productive) et l'émergence de la modernité (sous-entendu dans les relations sociales, les identités, les représentations). Cet auteur fait remarquer qu'en Amérique latine, les processus de modernisation techniques et économiques, qui peuvent être extrêmement rapides et importants, ne sont malheureusement le plus souvent pas accompagnés par l'introduction de relations sociales et politiques plus modernes (égalité des femmes, émergence de la citoyenneté et de la participation, dépassement du clientélisme et du paternalisme, etc.). Il en résulte que des formes sociales anciennes voire archaïques sont souvent " habillées » avec de nouveaux objets techniques, de nouveaux modes de gestion, un nouveau vocabulaire... Qu'en est-il de l'agriculture familiale ?

En Argentine, dans les discours de la presse, de la population et même de la littérature scientifique, la modernisation technique, mais aussi la modernité sociale et culturelle, sont présentées comme essentiellement portées par une classe émergente d'agriculteurs entrepreneurs, pampéens mais pas uniquement (Reboratti, 2005 ; Hernández, 2009). "L'agriculture familiale » apparaît ainsi (depuis l'émergence d'un nouveau discours dans les années 2000) comme une revendication légitime et importante, mais dans le même temps elle est reliée non seulement à des formes anciennes, voire archaïques, de production mais aussi à des rapports sociaux et des cultures "traditionnels ». Cette vision passéiste est encore plus forte lorsqu'il est fait mention aux agricultures familiales extrapampéennes qui sont le plus souvent désignées par le terme "d'agricultures paysannes ". Dans cette recherche, nous souhaitons montrer l'inventivité et l'innovation sociale et politique portée par des 
populations dites paysannes ou traditionnelles, un caractère particulièrement innovant qui est masqué non seulement par les clichés que nous venons de présenter, mais aussi par les propres discours des intéressés qui mettent en avant, dans leur revendication sociale et culturelle, l'importance de la " tradition » et du "patrimoine ", et donc leurs liens au passé. Il est d'autant plus important de mettre en valeur cette dimension du mouvement récent de "l'agriculture familiale " que, comme le fait remarquer Albaladejo (2009), la modernisation technique et économique en Amérique Latine et les discours nouveaux qui l'ont accompagné ont le plus souvent habillé des formes socioculturelles anciennes qui perdurent. L'agriculture familiale serait donc porteuse d'une possible rupture dans les transformations des mondes ruraux en Argentine.

À travers trois exemples pris dans les trois grandes régions d'agricultures paysannes de l'Argentine (le Nord-Ouest, le Nord-Est et la Patagonie, Fig. 1), nous avons pu démontrer que le mouvement de l'agriculture familiale peut être l'émanation d'une forte modernisation sociale, politique et culturelle, même si, dans les cas présentés et à l'inverse de ce qui a été observé pour l'histoire de la modernisation agricole en Amérique latine, celle-ci ne va pas toujours de pair avec une modernisation technique et économique.

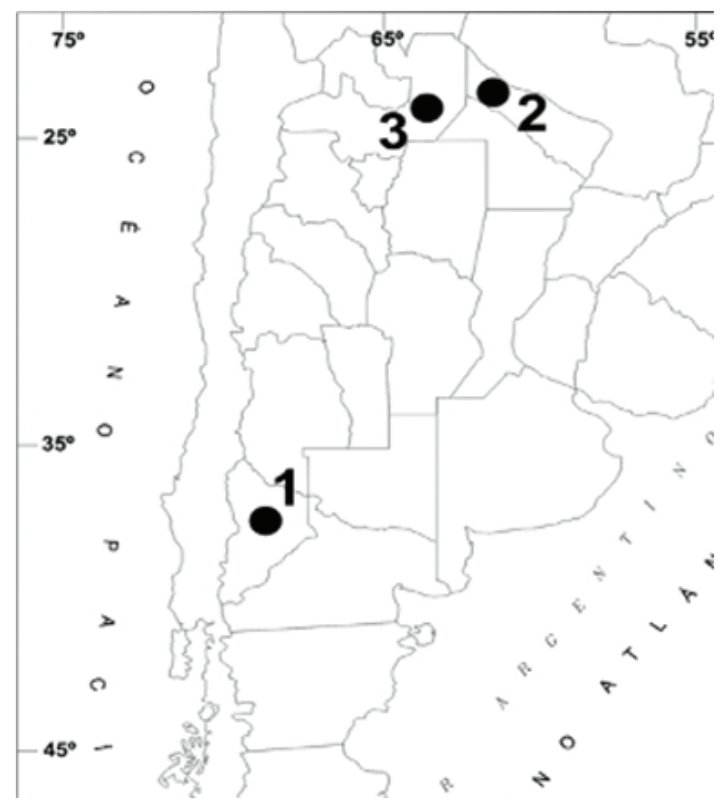

Légende:

1 - Zone centrale de la province de Neuquén

2 - Chaco Formoseño

3- Chaco Salteño

Fig. 1. Situation des trois cas analysés en Argentine. 


\section{Trois situations d'agricultures familiales}

\section{Cas I. L'action collective et l'organisation des petits producteurs ovins et caprins de la zone centrale de la province de Neuquén (Nord de la Patagonie)}

Le territoire de la Patagonie argentine, et tout particulièrement de la province de Neuquén, présente de forts contrastes de développement entre les zones irriguées des vallées et les zones des plateaux ou steppes.

Après la période d'expulsion et d'extermination des populations autochtones à la fin du XIX ${ }^{\mathrm{e}}$ siècle, la région est progressivement repeuplée par une population issue du métissage des descendants des peuples mapuches et tehuelches et de la population créole. Ce nouveau peuplement s'approprie cet espace marginal en consolidant un mode de vie original basé en grande partie sur l'élevage extensif. Ces éleveurs gardent des modes sociaux et techniques d'organisation hérités de la période coloniale, mais toujours marqués par la pauvreté. De plus, ils se retrouvent dispersés sur un immense territoire. Les activités économiques dominantes ont été, et sont encore aujourd'hui, la petite agriculture et l'élevage extensif, en particulier des moutons et des chèvres. C'est autour des pratiques traditionnelles de l'agriculture que se produit aujourd'hui un processus organisationnel visant à améliorer la situation des familles rurales. Cependant, ce n'est qu'avec l'intervention de l'État, à travers de nouveaux projets de développement et des incitations juridiques, que commence un processus d'apprentissage collectif et émergent de nouvelles formes d'organisation. Celles-ci concernaient initialement des activités spécifiques à la production et à la commercialisation puis se sont progressivement étendues à d'autres activités. Les processus de formation d'assemblées locales, l'émergence de nouvelles formes de leadership notamment à travers des élections de représentants et de délégués, la reconnaissance des organisations au sein des institutions locales, se traduisent par des formes d'action qui tendent à faire émerger de nouvelles formes d'organisations et une nouvelle représentation de la vie en société.

Les paysans pauvres désignés sous les termes de "crianceros " et "puesteros " (ces derniers s'occupant d'animaux en pension d'autres éleveurs) représentent la forme d'agriculture largement dominante dans le Nord-Ouest de la Patagonie : ils sont plus de 7500 selon Bendini, et al. (2002). Ils représentent $90 \%$ de la population rurale de la zone nord et du centre de la province de Neuquén. Localement le terme "crianceros " désigne l'ensemble des producteurs familiaux aux caractéristiques paysannes prononcées et se consacrant essentiellement à l'élevage extensif d'ovins et caprins. Plus précisément, le territoire sur lequel les «crianceros " réalisent leurs activités s'étend du sud de la province de Mendoza jusqu'au centre de la province de Chubut. Toutefois, ce sont les provinces de Neuquén et de Rio Negro qui concentrent les plus fortes densités de «crianceros». Cette zone comprend les vallées d'altitude de la cordillère et la précordillère des Andes, recouvertes de forêts humides et les steppes arides du plateau patagon. Pour la majorité des petits producteurs du pays résidant dans des zones peu peuplées et isolées, l'action collective organisée 
constitue l'une des stratégies pour surmonter la faible structuration du secteur. La description du processus historique de consolidation de l'Association d'éleveurs de chèvres angora de Neuquén (ACCAN) permet de comprendre les processus d'autonomisation et de renforcement des capacités d'action de l'ensemble des producteurs se trouvant dans des situations similaires ${ }^{11}$. L'ACCAN est une organisation sans but lucratif née en 1998 qui compte 70 familles de petits producteurs (Sapag, 2008). L'introduction de la race angora dans la région, ainsi que dans les différents projets d'assistance technique qui aboutirent à la création du programme Mohair, est à l'origine de l'organisation. Le programme dépend du ministère de l'Agriculture, de l'Élevage et de la Pêche et intègre plusieurs comités provinciaux composés de représentants des producteurs et des institutions. L'objectif du programme était initialement l'amélioration génétique et le renforcement de la connaissance du produit et de sa commercialisation.

Le mohair est une laine spéciale, plus large, douce, brillante et résistante que la laine commune. Elle est issue de l'élevage des chèvres de race angora et son prix au producteur est établi en fonction de sa qualité. La production est réalisée individuellement mais le stockage, la certification et la commercialisation sont conduits au sein de collectifs de producteurs. En effet, chaque producteur pris individuellement ne produit pas une quantité suffisante pour justifier le ramassage par des négociants. Après un premier tri réalisé par le producteur, intervient la certification par l'Association. Bien qu'il dialogue avec les producteurs, le certificateur est souverain dans ses décisions. L'ensemble de ce processus organisationnel repose sur des apprentissages individuels et collectifs qui correspondent à une innovation discrète ${ }^{12}$. Ce type de certification participative représente une forme de capital social et repose sur une organisation locale délibérative, ce qui est totalement innovant dans l'histoire du groupe concerné. L'ACCAN a connu dans son passé une cohésion forte faite de relations de confiance et de savoir-faire, notamment pour l'organisation de la tonte au ciseau. Cette activité, réalisée par l'ensemble des membres chaque année, était l'occasion d'échanger et de s'informer sur les possibilités d'amélioration du processus de production et de commercialisation. Les éleveurs firent ensuite peu à peu appel à des techniciens extérieurs au groupe, notamment des agents de développement de l'INTA. Puis ils prirent contact avec d'autres organisations ainsi qu'avec d'autres acheteurs que les commerçants locaux avec qui ils sont liés par des relations sociales traditionnelles (parenté symbolique, paternalisme, etc.). La complexité des activités de commercialisation, ainsi que celle de la logistique requise, ont été à l'origine d'une pratique collective qui s'appuie sur des formes de relation participatives et délibératives entre les membres du groupe et sur d'importants réseaux de dialogue

11. Dans la même Province de Neuquén, Perez Centeno (2007) a aussi étudié le processus organisationnel conduisant à la valorisation de la chèvre créole.

12. Une " innovation discrète " selon Albaladejo (2005) est un processus d'innovation en dehors des modèles dominants de modernisation en agriculture qui peut être apparemment très " modeste " mais qui de fait contribue à faire émerger de nouvelles formes de relation au territoire, à l'État et au marché autrement dit de nouvelles médiations territoriales. Elle est donc plus qu'une résistance ou une innovation de survie ou de résilience. 
techniques qui permettent de répondre aux demandes du secteur. L'expérience sociale de ces nouvelles formes d'être en société est, dans le cadre de ces groupes basés sur la tradition, une innovation considérable. Reliée à de nouvelles pratiques des agents de l'État, à de nouvelles formes de commercialisation et de nouveaux objets techniques (machines à tondre, parcs) impliquant de nouvelles formes de relation au territoire, cette expérience inédite est plus qu'une étrangeté ou une innovation induite par des programmes de développement : elle est une nouvelle forme de médiation territoriale et donc une innovation discrète (Albaladejo, 2005).

Le choix de cette étude de cas, présentée de manière résumée, est justifié par la dynamique d'apprentissage et d'accumulation de capital relationnel. De plus, elle rend compte de l'importance des actions de l'appareil politique et de développement favorisant ces processus socio-organisationnels. Ces pratiques ont permis l'émergence d'une forme plus transparente de leadership, et surtout un processus d'auto-identification des individus en tant que sujets de droits qui expriment leurs besoins et leurs demandes. Ce processus d'apprentissage individuel et collectif peut être interprété en termes de capital social renforçant des réseaux sociaux au sein et à l'extérieur des localités rurales : parajes et comunidades. La création l'ACCAN, l'élaboration de son statut juridique, la formation du réseau des acteurs, et la définition des rôles dans l'organisation, ont été des moments clé du processus d'organisation et de construction d'une nouvelle territorialité, autrement dit d'une construction du territoire non plus seulement par le groupe social en tant que tel mais aussi par les pratiques de l'individu transformé en " sujet " selon Albaladejo (2005 et 2009). Cette nouvelle capacité de participer se transfere dans d'autres domaines de l'action territoriale et permet de revendiquer des appuis non seulement dans la sphère productive mais aussi dans le domaine de la santé, la communication et la représentativité politique.

\section{Cas II. La population créole du Chaco Formoseño : de la résistance à l'innovation}

La zone étudiée, située à l'ouest de la province de Formosa, appartient à la région phytogéographique du Parque chaqueño semi-aride qui comprend les départements Matacos, Ramón Lista et Bermejo (Adamoli et al. 1972). La population de l'Ouest de Formosa se compose principalement d'indigènes (ethnies Wichí, Tobas et Pilagá) et de créoles, ces derniers étant en grande partie responsables du développement des activités agricoles dans la région. La population créole est issue des familles d'éleveurs originaires de Salta et de Santiago del Estero qui, au début du XXe siècle, se sont installés dans la région à la recherche de terres pour y faire pâturer leurs animaux. Ces familles ont connu une période de prospérité économique, mais leurs systèmes de production peu durables ont provoqué en quelques décennies une forte dégradation des ressources naturelles du fait du surpâturage (Sanz, 2010).

Ce processus de dégradation de l'environnement a entrainé un déclin de l'agriculture : avec une plus faible productivité des systèmes, l'absence de diversification des systèmes fourragers basés seulement sur la valorisation du fourrage xérophile des 
forêts dégradées, une population rurale pauvre et en diminution, des infrastructures routières précaires et forte dépendance vis-à-vis de la politique économique de l'État et de la Province. À partir des années 1970, la situation s'est aggravée. Tout d'abord, la plupart des troupeaux ont été touchés par la rage ; 30 à $40 \%$ des troupeaux ont été décimés. Ensuite, dans les années 1990, le processus de désengagement de l'État s'est traduit par la fermeture définitive du chemin de fer, ce qui a renforcé l'isolement des éleveurs. Une route goudronnée ne sera construite qu’en 2004.

Ainsi, la majorité des familles créoles rurales, les descendants des éleveurs qui ont colonisé le territoire, se retrouve de plus en plus isolée dans leurs exploitations dénommées "puestos" (habitat dispersé), tandis que d'autres familles se sont regroupées au sein des "parajes" (hameaux), par groupes de quatre familles ou plus qui partagent un terrain à proximité d'une école primaire. Ce type de peuplement contraste fortement avec celui de l'Est de Formosa, où les conditions sont plus favorables aux cultures et où la densité de peuplement est nettement supérieure.

La principale activité économique des familles est l'élevage de bovins et de chèvres sur des espaces ouverts en prairies naturelles ou sur des espaces forestiers indigènes dégradés. Les installations d'élevage sont très précaires (parcs, couloirs de contention, etc.) et la gestion du troupeau rudimentaire. Le niveau de revenu familial est très faible, provenant presque exclusivement de la vente de bétail et de l'apport des programmes nationaux de l'aide sociale. Il est important de noter que l'Ouest du territoire de Formosa se compose d'environ $99 \%$ de petits producteurs, qui détiennent $92 \%$ des bovins (Sanz, 2010) et que $80 \%$ des producteurs ont moins de 50 vaches. L'isolement des familles créoles et leur éloignement géographique les unes des autres a favorisé un comportement de repli sur la famille proche (parfois stigmatisé par le terme "d'individualisme ») qui s'est ancré dans la culture de ces populations, limitant ainsi la possibilité de générer des formes d'associations plus larges. Cette situation a aussi permis de mettre en valeur les compétences et croyances locales, notamment les traditions culturelles créoles liées aux chevaux, pour survivre dans cet environnement difficile.

\section{Construire des organisations paysannes sur le territoire}

En dépit de la situation structurelle décrite, les travaux de recherche réalisés ont clairement montré que, dans cette région, ont eu lieu, au cours des dernières décennies, des processus qui different de l'image d'immobilité habituellement attribuée à ces agriculteurs (Sanz, 2010). Ces processus ont été initiés par une intervention plus active du secteur public, qui s'est exprimée d'abord timidement dans les années 1990. Dans une première étape, l'INTA a développé le programme Prohuerta (Cittadini, 2012) et le SENASA ${ }^{13}$ a aidé à la constitution d'une commission pour la mise en œuvre d'un plan de vaccination. Ces interventions ont été consolidées à partir de 2003, avec la mise en œuvre par l'INTA d'une approche territoriale du développement et de la promotion de l'organisation locale, et avec la mise en

13. Service national de santé et qualité des aliments. 
œuvre par la Province de programmes visant à promouvoir les petits agriculteurs $\left(\mathrm{PAIPPA}^{14}\right)$ et des actions visant à régulariser la propriété foncière. En effet, les producteurs avaient en général un titre de possession de fait de leurs terres, mais ils n'en avaient pas le titre de propriété légal. Il faut aussi souligner l'action d'une $\mathrm{ONG}^{15}$ locale liée à l'Église catholique. La capacité d'action des producteurs, stigmatisés comme " réfractaires au changement et individualistes ", a pourtant donné lieu à l'émergence de 18 organisations de producteurs constituées en moins de dix ans.

Différents conflits ont induit à l'émergence de ces nouvelles constructions sociales, appelées " associations " ${ }^{16}$, concernant l'occupation des terres entre les Créoles et les peuples originaires ou encore la propriété des terres entre les paysans et une entreprise privée. Cette situation est aussi le résultat de l'absence de politiques provinciales qui appuient la gestion et la légalisation de l'occupation des terres de l'ouest de Formosa, ainsi que de l'arrivée de puissants investisseurs immobiliers sur le territoire.

Les organisations de producteurs mentionnées ci-dessus sont distribuées sur l'ensemble du territoire et reçoivent une assistance technique et organisationnelle par des institutions gouvernementales et non gouvernementales. Cet appui concerne différents problèmes, tels que l'accès aux titres de propriété foncière, la faible production de l'élevage, ou le manque d'expérience de l'organisation, entre autres. Dans ce contexte, le plan de colonisation de l'Ouest de Formosa mis en place par le Gouvernement provincial, a pour objectif de régulariser le statut juridique des terres publiques et de fournir des ouvrages hydrauliques (barrages) pour un accès sécurisé à l'eau pour la plupart des exploitations familiales du territoire.

Ainsi, les conflits et les incertitudes liés à la propriété foncière ont été les principaux facteurs qui ont conduit à l'action collective des paysans. Cependant, sans les relations de solidarité qui se sont structurées entre les familles d'éleveurs, la construction d'une action organisée et durable n'aurait pas été possible. Par ailleurs, toutes les associations reposent sur une identité commune, construite sur la base des traditions et de la condition sociale partagées par l'ensemble des membres. L'identité commune est un des fondements de l'unité des participants dans l'action collective : plus cette identité commune est forte dans le groupe, plus il a été facile de construire une unité durable (Pineiro, 2004). Ainsi, le fait d'être créole entretient un fort sentiment d'identité commune qui est renforcé par la condition d'éleveur, de sans terre ou d'occupant précaire de la terre et finalement par la condition d'appartenance à une région ou un lieu particulier.

En se regroupant pour lutter pour l'accès aux titres de propriété, les producteurs ont pris conscience de leur rôle d'acteurs sur le territoire et de leur pouvoir dans un conflit de territorialité. Cela a aussi favorisé la valorisation d'un projet original de vie et de production. Ils ont amélioré l'infrastructure des clôtures et ont participé

14. Programme d'aide intégral au petit producteur.

15. Organisation non gouvernementale : Fondation Angela Garzon.

16. Il s'agit des d'associations civiles, souvent sans statut juridique, mais qui permet aux producteurs de se réunir et s'organiser pour lutter pour ses besoins. 
à la recherche d'améliorations techniques appropriées à leur contexte, comme les technologies d'approvisionnement en fourrage adaptées à leurs systèmes, technologies mises au point en collaboration avec l'agence locale de l'INTA. Ils ont aussi pris conscience de l'importance de certains produits traditionnels qui n'étaient pas très reconnus, comme le "charque $"{ }^{17}$. Ainsi, une association de producteurs a installé une usine dans le village pour améliorer la commercialisation locale du bétail. Mettant en pratique l'écoute des autres et un travail participatif, des agents de développement ont pu évaluer l'importance de certaines activités locales dites " traditionnelles » qui, bien qu'étant secondaires, ont joué et continuent de jouer un rôle important et stratégique dans l'organisation et la durabilité du système productif. C'est le cas notamment de la production de fromage. Le fromage " créole » est un sous-produit de l'activité d'élevage. Il représente cependant un apport décisif à l'économie de la famille, car il constitue un apport d'argent durant trois à quatre mois dans l'année. Il est également une activité structurante d'un ensemble de pratiques de production du système de gestion de l'élevage (Sanz, 2010). Ce processus de "modernisation " chez les paysans de l'Ouest de Formosa a été rendu possible grâce au travail des agents de développement qui ont su dépasser les conceptions de la modernisation en termes de simple "transfert de technologie " et se sont dotés de concepts et d'outils (approche territoriale et développement participatif, etc.) facilitant les capacités d'expression de ces communautés et respectant les logiques et conditions de leurs systèmes de production (Cittadini, 2013). Les changements ne sont donc pas uniquement à chercher dans les pratiques locales mais aussi dans les pratiques des institutions et notamment des institutions de développement (comme le propose la notion d'innovation discrète introduite par Albaladejo, 2005).

\section{Cas III. Les habitants créoles du Chaco Salteño: innover pour résister}

Le développement du modèle de l'agrobusiness en Argentine a provoqué d'importantes transformations dans les systèmes de production, les écosystèmes et les structures sociales qui étaient traditionnellement intimement liées au milieu naturel dont elles sont originaires et où elles se sont développées historiquement. Dans ce contexte, l'avancée du front agricole, du soja en particulier, dans le Nord-Est du pays est problématique et engendre d'importantes modifications dans le monde rural.

Les communautés paysannes du territoire du Chaco Salteño dans le Nord-Est de la province de Salta illustrent précisément ces processus. En termes de peuplement, la zone présente une densité relativement faible ainsi qu'une diversité ethnique et culturelle qui façonne une trame sociale complexe. Les agriculteurs familiaux sont représentés sur le territoire par deux groupes sociaux principaux : les communautés indigènes et les habitants créoles qui se sont installés, suite à des migrations internes

17. La viande salée et séchée au soleil 
depuis la moitié du XIX ${ }^{e}$ siècle, en pérennisant et transformant leurs coutumes, cultures et identités d'origine.

Les problématiques liées à l'avancée du front agricole, aux disputes autour du foncier et les tensions au sein du territoire affectent les deux populations. Cependant, la présente étude se concentre sur les populations créoles du Chaco Salteño. Après avoir présenté les processus d'expansion du front agricole du fait de l'essor des entreprises capitalistes, nous analyserons la manière originale par laquelle les producteurs familiaux ont réagi, dans une dispute de territorialité.

\section{L'expansion du front agricole dans le Chaco Salteño}

Dans les années 1960, le prix du haricot a augmenté sur le marché international favorisant sa production dans le Sud de la province de Salta. Ainsi, le Sud-Est de la province est entré sur le marché agroexportateur avec comme conséquence la déforestation et l'acquisition de terres à bas prix par de grands exploitants pratiquant l'agriculture extensive. Jusqu'aux années 1980, ces terres furent dédiées à la culture de haricots même si le recensement national agricole de 1988 révélait déjà le récent essor d'une autre culture dans la région : le soja.

Le rapide développement de la production de soja en Argentine est remarquable, cette culture pratiquement méconnue dans les années 1970 est devenue dans les années 1990, la première production nationale (Reboratti, 2006). En effet, la deuxième étape de l'expansion agricole a été marquée par une augmentation continue des cultures au nord comme à l'est de la région. En dix ans, les prix du foncier ont pratiquement triplé, accentuant la concentration de la terre dans les mains d'un petit groupe de grands producteurs et d'entreprises soutenus par des capitaux extérieurs.

Actuellement, l'extension des surfaces agricoles semble avoir atteint sa limite du fait des conditions de sécheresse trop contraignantes pour l'implantation de la majorité des grandes cultures sur ces territoires. Cependant, il est possible que dans quelques années nous assistions à l'apparition de variétés tolérantes ou résistantes au stress hydrique. Des études sont actuellement conduites pour mettre au point des variétés basées sur des gènes de résistance à la sécheresse qui pourraient constituer une alternative pour les grands producteurs, mais qui engendreront aussi une pression considérable sur les surfaces encore en forêts et sur les espaces des agricultures paysannes.

\section{Le processus d'action collective dans le Chaco Salteño}

Les transformations de l'appareil productif régional menacent les modes de production et de reproduction des peuples créoles. Mais celles-ci renforcent aussi chez eux, simultanément, un fort sentiment d'appartenance au territoire local en tant que lieu les définissant et les caractérisant historiquement et socialement. Les agriculteurs familiaux qui, à un moment donné et dans un espace donné, ont adopté des modes de production et des formes d'organisation correspondant à leurs stratégies 
de reproduction sociale, ont été obligés, suite à ce changement de contexte, de créer de nouveaux modes de vie, de nouveaux objectifs et de nouvelles formes d'adaptations.

Les cultures et les identités paysannes s'expriment en particulier dans le milieu discret et privé de la vie quotidienne des familles créoles. Ainsi il est possible de considérer cette préservation et recréation de pratiques et éléments de vie quotidienne comme une forme de résistance conçue dans l'espace et dans le temps de la quotidienneté. Cependant, même si ces éléments contribuent de manière importante aux formes de reproduction socioculturelle des populations, ils n'assurent pas de perspectives de pérennité sur le long terme. En particulier, ils ne peuvent constituer à eux seuls une alternative permettant d'affronter les problématiques qui portent atteinte à la persistance d'un groupe social de manière durable et ancré dans un espace territorial.

La situation est différente quand les pratiques qui se sont préservées à travers une résistance discrète voire passive, se voient articulées avec de nouvelles stratégies se matérialisant au travers de pratiques collectives dont l'objectif et d'augmenter le capital social (Bourdieu, 2001). Ces pratiques collectives peuvent être perçues comme innovatrices ou comme des formes d'adaptations à l'origine d'un projet collectif transformateur qui constitue une certaine opportunité d'innovation en termes de changement social. C'est précisément ce que nous pouvons observer dans l'étude que nous avons réalisée.

Les prémices de l'organisation se trouvent dans la création de groupes traditionalistes ou "Centres gauchos ", présents sur l'ensemble de la province depuis le milieu du XX ${ }^{\mathrm{e}}$ siècle (Hernandez, 2013). Ils sont en principe fortement liés à l'action culturelle et traditionaliste, et non à l'action collective qui vise un changement social ou une réaction à un problème commun. Leurs objectifs étaient la préservation et la revalorisation de la culture créole, le fonctionnement quotidien de lieux de rencontre et de loisirs, ainsi que l'organisation de festivités et de manifestations patriotiques, religieuses, culturelles. L'importance de ces groupes est indéniable puisqu'ils précèdent les formes actuelles d'organisation collective. Ils représentent les premières expériences de participation dans des espaces formels de réunion pour la plupart des leaders et des participants des organisations actuelles.

D’autres espaces de participation pour les éleveurs ont aussi été créés pour organiser des campagnes de vaccination, la distribution d'intrants et la mise en œuvre des procédures de régularisation des troupeaux de bétail en vue des contrôles officiels sanitaires. Ce sont les centres d'élevage mis en place dans de nombreux villages du Chaco Salteño. Dans ce cas, l'intérêt commun pour l'élevage bovin a constitué un socle pour la constitution de l'organisation des producteurs créoles. Les premières organisations locales, les Centres gauchos et les centres d'élevage, ont été construites autour d'intérêts proches du quotidien de la population créole à savoir la culture, l'identité et l'élevage traditionnel.

Les centres d'élevage se sont positionnés comme bénéficiaires des projets et des programmes (crédits, subventions, assistance technique) de soutien à l'élevage, et ont ainsi renforcé leur rôle, en assumant des fonctions supplémentaires et en 
devenant les premières organisations de producteurs locaux insérées dans le territoire. Toutefois, il convient de souligner que la création de centres d'élevage, a également coïncidé avec l'avènement des processus de transformation territoriale et le durcissement des conflits fonciers, conséquence de l'avancée progressive de la frontière agricole. Avec les centres d'élevage émergent les premiers efforts conjointement aux institutions gouvernementales pour conseiller, soutenir et répondre aux préoccupations des producteurs. À la fin des années 1980, dans certaines régions du Chaco Salteño, a émergé la première action collective organisée pour revendiquer le droit sur la propriété foncière. Ainsi se sont formés des groupes ou des comités au niveau des localités. Ensuite, des groupes au niveau régional se sont formés avec les délégués des groupes locaux. Après avoir atteint une notoriété suffisante, les organisations paysannes ont progressivement pu accéder à un espace institutionnel et devenir des interlocuteurs reconnus des différentes instances gouvernementales. Cela était impensable seulement quelques années auparavant. Ils ont pu avoir une action directe sur des questions centrales, non seulement territoriales mais de niveau régional et national, telles que le foncier, la déforestation incontrôlée, l'avancée de la frontière agricole, les politiques publiques pour l'agriculture familiale, la planification de l'utilisation des terres et le cadre législatif actuel. Parmi les principales actions et les événements les plus marquants, nous pouvons mentionner la mobilisation collective pour empêcher l'expulsion de familles créoles, la participation et l'organisation de manifestations et d'événements publics. Un exemple concret est le dépôt, en 2008 par les dirigeants locaux, d'un recours devant la Cour suprême de justice de la Nation et l'obtention de la cessation temporaire de la déforestation autorisée par la province de Salta depuis fin 2007.

En 2010, pour préserver les producteurs des expulsions et affirmer la « fonction sociale de la terre ", un projet de loi a vu le jour. Élaboré conjointement avec d'autres organisations paysannes du pays, il a été adopté par l'Assemblée législative de la province de Salta et a eu un impact très fort dans la région. L'état d'urgence territoriale a été déclaré pour une période de cinq ans, afin de garantir la possession des terres rurales qu'ils occupent. Cette déclaration a été approuvée par le Forum pour l'agriculture familiale (FONAF), le Mouvement national paysan et indigène (MNCI, affilié à Via Campesina), le Front national paysan (FNC), le Mouvement paysan de libération (MCL) et les organismes provinciaux comme la " Mesa de la Agricultura Familiar » à Buenos Aires.

Immédiatement, la plupart des organisations paysannes constituées ou en cours de constitution, soit plus de vingt organisations regroupant plus de 500 familles créoles, se sont regroupées au sein d'une nouvelle entité dotée d'une large représentativité, la Fédération des producteurs familiaux. Celle-ci a rapidement réussi à se positionner dans un plus grand espace de participation au niveau national. À l'échelle territoriale et provinciale, l'histoire la plus récente d'action collective est la présentation du projet de loi «Felipe Burgos Développement rural pour une agriculture familiale ", une loi conçue avec et pour les agriculteurs et les populations autochtones de Salta. Les objectifs de ce projet de loi visent une meilleure qualité de vie, le renforcement des droits, l'accès à des moyens pour renforcer la productivité et un meilleur accès aux marchés pour la population rurale et originaire de Salta. 
Pour résumer, au cours de la dernière décennie, la création et la participation à des organisations d'agriculteurs sont devenues, face aux transformations territoriales, les formes les plus efficaces et les plus visibles d'adaptation collective des Créoles du Chaco Salteño. Les compétences acquises et le repositionnement dans l'espace social réalisé au cours du processus, ont mis en évidence la capacité d'adaptation collective pour mener des actions organisées très pertinentes et d'importance territoriale et régionale.

\section{Discussion et conclusion}

Les différents cas que nous avons étudiés dans trois contextes régionaux différents présentent des éléments communs notamment en ce qui concerne les processus organisationnels locaux liés à la lutte pour la propriété foncière. De plus, dans les trois cas, ces processus organisationnels particuliers émanent de la population paysanne et des histoires sociales locales, et ils ont été consolidés par l'action de programmes d'intervention de l'État en appui aux communautés paysannes ayant réussi à s'organiser de façon formelle sur la base de dynamiques participatives. Les litiges sur le territoire et/ou la confrontation des projets territoriaux sont aussi fortement présents dans les trois études de cas que ce soit de manière ouverte ou latente. Selon la théorie des transformations territoriales de Raffestin (1986, 1987), une théorie mobilisée et adaptée par Albaladejo (2004), on entend par "territoire " une série de liens conceptuels et matériels entre les sociétés et leurs espaces. Selon Raffestin, tout processus qui implique une construction de sens dans les relations au territoire, à travers notamment des dispositifs organisationnels et matériels (processus baptisé " reterritorialisation »), s'accompagne nécessairement d'une destruction de l'ordre social, politique et technique précédent, qualifié de "déterritorialisation ». De fait, chacune de ces formes d'agriculture familiale émergentes produit un type spécifique de territoire avec ses caractéristiques et ses logiques propres. Il n'est ainsi pas possible de parler "d'une " agriculture paysanne ou familiale en tant que type normé d'activité agricole qui servirait de référence pour tout le pays. En revanche, les processus sociaux et territoriaux sur lesquels prennent appui ces formes d'agriculture peuvent être comparés. En premier lieu, la présence de situations de conflit avec d'autres formes d'agriculture ou d'autres formes d'occupation du territoire est un élément contextuel commun aux trois situations, et un facteur important de production de nouvelles formes organisationnelles qui ne se présentent pas forcément en continuité avec les formes sociales traditionnelles. Ces situations de conflit sont certes plus explicites, voire violentes, dans le Nord de l'Argentine, alors que dans le Sud le conflit est plus latent, ce qui est en partie explicable par la dispersion géographique propre aux zones de faible densité de population. Dans les trois cas présentés, les conflits, les tensions sociales avec d'autres groupes d'intérêt, ont finalement constitué un contexte d'apprentissage de nouvelles formes de représentation et d'organisation. À une autre échelle, ces situations de confrontation entraînent des actions structurantes au niveau national ou provincial, comme le montre le plan PAIPA qui offre en retour, au niveau local, une personnalité juridique et des espaces de 
représentation avec la délégation de la parole et l'accès à des cercles qui ne seraient pas accessibles sans le processus organisationnel développé.

Cette transformation de l'espace a mis en évidence de nouvelles territorialités, c'està-dire différentes formes de perception, d'évaluation et d'appropriation de certaines zones géographiques par les différents acteurs (Montañez Gomez et Mahecha Delgado, 1998), qui dans ce cas sont exprimées par les Créoles, les aborigènes, les investisseurs agricoles et l'État (provincial et national). Avec Fernandes (2008), nous pouvons dire que ces territorialités ont été découvertes quand le territoire a été envisagé comme un ensemble, avec ses différentes échelles multidimensionnelles, et que ses différentes utilisations ont commencé à se faire reconnaître.

À l'ouest de Formosa, la revendication d'un territoire pour l'agriculture paysanne peut être considérée comme un phénomène nouveau et innovant, porté par des formes d'organisation et de leadership qui ne sont en rien des survivances de formes sociales locales traditionnelles. Ce processus était généré en partie comme une réaction face à des situations totalement nouvelles de confrontations, produisant des conflits et des incertitudes qui ont conduit la paysannerie locale à mobiliser de nouvelles formes d'action collective pour défendre ses droits (Tarrow, 1997). Dans de nombreux cas, l'action collective des groupes de paysans s'est transformée en un système organisé, en créant de nouvelles constructions sociales voire légales appelées " associations ". Ces nouvelles constructions sociales se superposent ou s'articulent de façon complexe aux formes sociales antérieures dans un schéma de coprésence entre des formes sociales très anciennes liées notamment aux habitus des agents et de nouvelles et innovantes expériences. Martins (1999) a pu décrire cette juxtaposition complexe du moderne et du traditionnel au Brésil dans une figure théorique qu'il a nommée "l'histoire lente » autrement dit une histoire qui hybride continuellement le nouveau et l'ancien.

Ce ne sont pas seulement les conflits et les confrontations qui expliquent ces apprentissages sociaux. Dans d'autres cas en effet, la présence d'organismes gouvernementaux, des ONG, et l'action des gouvernements provinciaux ont constitué un autre facteur d'une importance considérable et ont favorisé aussi l'émergence de nouvelles organisations paysannes sur le territoire.

Dans tous ces cas, les agriculteurs familiaux sont très loin de l'image de l'inertie ou de traditionalisme par laquelle ils sont souvent dépeints. Au contraire, ils sont les protagonistes actifs de procédés modernes. De plus, dans la plupart des cas, les organisations des agriculteurs et les agents de développement qui leur sont liés ont cherché à éviter les conséquences négatives des processus habituels de la modernisation imposée, une modernisation qui faisait fi de la culture locale et des spécificités de développement.

Nous observons dans les différentes régions du pays des processus collectifs par lesquels se sont initiées et consolidées des alternatives locales innovantes qui ont été à même de se relier à un processus de visualisation et de légitimation au niveau national ou provincial. En effet, un mouvement au niveau national et un secteur de l'État ont permis l'émergence d'opportunités et de propositions de changement en faveur de groupes sociaux locaux qui auraient eu du mal à se consolider sans ce relais. Le mouvement de l'agriculture familiale au niveau national a favorisé, ainsi 
localement, des capacités d'adaptation et de réinvention collective, celles-ci ayant trouvé leur plus haute expression et visibilité dans l'augmentation progressive de la dotation en capital social des organisations locales. Par là même, grâce au travail constant d'auto-validation en tant que groupe social, de nouveaux leaders ont légitimé leurs pratiques et obtenu une place importante dans l'espace public local, en transcendant même les frontières de la communauté locale.

La participation active des institutions de l'État dans ces processus (Gisclard et Allaire, 2012), offre la possibilité de disposer d'une expérience importante pour une évaluation critique de l'impact de l'action institutionnelle et ainsi redéfinir des stratégies d'intervention. Ces expériences fournissent des éléments pour ré-légitimer le métier de l'agent de développement (Cittadini, 2013), en impulsant une réflexion critique et prospective sur leurs propres pratiques, les faiblesses et les nouvelles compétences requises par les dynamiques territoriales actuelles.

\section{Bibliographie}

Adamoli, J., Neumann, R., Ratier de Colina, A., Morello J., 1972. El Chaco aluvional salteño. Revista de Investigaciones Agropecuaria. INTA. Serie 3, Clima y Suelo, IX, 5, 166-237.

Albaladejo, C., 2004. Innovaciones discretas y reterritorialización de la actividad agropecuaria en Argentina, Brasil y Francia, en Albaladejo, C., Bustos Cara, R. (Eds.), Desarrollo local y nuevas ruralidades en Argentina, UNS-Departamento de Geografía - IRD UR102 - INRA SAD - Univ, Toulouse Le Mirail UMR Dynamiques Rurales, Bahía Blanca, Argentina.

Albaladejo, C., 2005. Une Argentine " discrète »... Repérage de nouvelles territorialités en région pampéenne à partir de parcours d'entrepreneurs issus de l'agriculture familiale. Le cas du district de Saavedra (Pigüé), Norois, Environnement-Aménagement-Société, 197, 7-22.

Albaladejo, C., 2009. Médiations territoriales locales et développement rural. Vers de nouvelles compétences d'accompagnement de l'activité agricole. Les agricultures familiales dans les transformations territoriales en Argentine, au Brésil et en France. HDR Habilitation à Diriger des Recherches, Géographie et Aménagement, UFR Sciences Sociales Département de Géographie, 304 p.

Albaladejo, C., Bustos Cara, R., 2010. Compétences, action publique et action collective dans le développement agricole localisé en Argentine, in de Sainte Marie, C., Muchnik, J. (Eds), Le temps des SYAL. Techniques, aliments et territoires, Éditions Quae, 227-244.

Arendt, H., 1994. La condition de l'homme moderne, Paris, Pocket.

Bendini de Ortega, M., Ksakoumagkos, P., Destefanoi de Monsalvo, B., 1985. El trabajo trashumante en la Provincia del Neuquen, Universidad Nacional del Comahue, Neuquén, Argentina.

Bourdieu, P., 2001. El capital social. Apuntes provisionales. Zona Abierta 94/95, Madrid, España. 
Bustos Cara, R., 2002. Los sistemas territoriales. Etapas de Estructuración y Desestructuración en Argentina, Anales de Geografia de la Universidad Complutense, 22, 113-129.

Cittadini, R., 2012. Limites et potentialités de l'agroécologie. Leçons d'une expérience à grande échelle : le programme PROHUERTA en Argentine, in Goulet, F., Magda, D., Girard, N., Hernandez, V., Sarandon, S., (Eds), Agroécologie en Argentine et en France. Regards croisés, Paris, éditions L'Harmattan.

Cittadini, R., 2013. Programas de desarrollo territorial rural. Las teorías y las prácticas del desarrollo, en Feito, C., et al. (Ed.), Contribuciones internacionales al desarrollo local y rural: hacia una agenda de investigación, Universidad Nacional de La Matanza, Argentina, 93-102.

Dominguez, D., 2005. Trashumantes : la resistencia como vitalidad, in Giarracca, N., Teubal, M. (Ed.), El campo argentino en la encrucijada. Estrategias y resistencias sociales, ecos en la ciudad, Alilanza Editorial, Buenos Aires, 295-321.

Fernandes, B. M., 2008. Territorios y Desarrollo Rural en América Latina. Curso de doctorado en estudios sociales agrarios, Universidad Nacional de Córdoba.

Giarracca, N., 1990. El campesinado en Argentina : un debate tardío, Desarrollo Económico, Buenos Aires, 94, 55-65.

Gisclard, M., Allaire, G., 2012. L'institutionnalisation de l'agriculture familiale en Argentine : vers la reformulation d'un référentiel de développement rural, Autrepart, 62, 201-216.

Hernández, V., 2009. La ruralidad globalizada y el paradigma de los agronegocios en las pampas gringas, in Hernández, V., Gras, C. (Ed.), La Argentina rural. De la agricultura familiar a los agronegocios, Biblos, Buenos Aires, 39-64.

Hernández, H., 2013. El avance de la frontera agrícola en el Chaco Salteño : Estrategias de adaptación de los pobladores criollos, Tesis de Maestría PLIDER. Facultad de Ciencias Agrarias de Balcarce, UNMDP, Argentina.

Hernández, H., Carricart, P., 2010. Expansión de la frontera agrícola en el Chaco Salteño. Estrategias de adaptación de los pobladores criollos : Resistencia, Permanencia, Cambio. II Congreso Internacional de Desarrollo Local, I Jornadas Nacionales en Ciencias Sociales y Desarrollo Rural, Universidad Nacional de La Matanza, Argentina.

INDEC. 1988. Censo Nacional Agropecuario.

INDEC. 2002. Censo Nacional Agropecuario.

Jong, G. D., Tiscornia L., 1994. El minifundio en el Alto Valle del Río Negro. Estrategias de adaptación, Universidad del Comahue, Facultades de Ciencias Agrarias y de Humanidades, Neuquén, Argentina.

Martins de Souza, J., 1996. O tempo da fronteira. Retorno à controvérsia sobre o tempo histórico da frente de expansão e da frente pioneira, Tempo Social, 8.

Martins, J.D.S., 1999. O poder do atraso. Ensaios de sociologia da história lenta, Hucitec, São Paulo.

Montañez Gómez, G., Delgado Mahecha, O., 1998. Espacio, territorio y región : Conceptos básicos para un proyecto nacional. Disponible en : http:// www.geolatina.com/files/MONTANEZ_y_DELGADO.1998,pdf. [Ultimo acceso : Enero de 2008]. 
Muller, H. 2011. Crece el uso de los cultivos transgénicos, AGROPOST, 114. CPIA - Consejo Profesional de Ingeniería Agronómica.

Murmis M., 1993. Productores andinos en crisis : estudios sobre una larga epopeya, in Grupo de Estudios Sociales A. (Ed.), Campesinado y trabajo trashumante en Neuquén, Univ. Nac. del Comahue, Editorial La Colmena, Buenos Aires, 11-18.

Pérez Centeno, M., 2007. Transformation des stratégies sociales et productives des éleveurs transhumants de la province de Neuquén et de leurs relations avec les interventions de développement, Doctorat TESC Temps Espaces Sociétés Cultures, filière doctorale ESSOR Études Rurales, mention Développement.

Piñeiro, D., 2004. En busca de la identidad : La acción colectiva en los conflictos agrarios de América Latina. Ed. Consejo Latinoamericano de Ciencias Sociales (CLACSO), Buenos Aires.

Reboratti, C., 1990. Fronteras Agrarias en América Latina, Geocritica, Cuadernos Criticos de Geografía Humana, 87.

Reboratti, C., 2005. Efectos sociales de los cambios en la agricultura, Ciencia Hoy, Revista de Divulgacin Cientifica y Tecnológica de la Asociación Ciencia Hoy, Buenos Aires, 15, 87, 52-61

Reboratti, C., 2006. La Argentina rural entre la modernización y la exclusión, en Geraiges de Lemos, A. I., Arroyo, M., Silveira, M. L. (Eds), America Latina : cidade, campo e turismo. CLACSO, San Pablo, Brasil.

Renard, J.-P., 1992. Population et frontières : problématiques et méthodes, Espace, Populations Sociétés, 10,2, 167-184.

Sanz, P., 2010. Procesos organizativosde los productores criollos del oeste de Formosa, Tesis Maestría PLIDER, Universidad Nacional de La Plata.

Sapag, M. A., 2008. Procesos de Desarrollo Territorial en áreas escasamente pobladas. Forma de construcción de liderazgo y capital social en una organización de pequeños productores. Tesis de Maestría en procesos Locales de innovación y desarrollo rural (Plider), Facultad de Ciencias Agrarias y Forestales, Universidad Nacional de La Plata Julio 2008. 145 pag.

Schiavoni, G., 1995. Organización doméstica y apropiación de tierras fiscales en la frontera de Misiones (Argentina), Desarrollo Económico, Revista de Ciencias Sociales, 34, 1363, 595-608.

Tarrow, S., 1997. El poder en movimiento - Los nuevos movimientos sociales, la acción colectiva y la política, Ed. Alianza. Madrid.

Teruel, A., 2005. Misiones, economía y sociedad. La frontera chaqueña del Noroeste Argentino en el siglo XIX, Universidad Nacional de Quilmes, Buenos Aires, Argentina.

Zusman, P., 1999. Representaciones, imaginarios y conceptos en torno a la producción material de las fronteras. Reflexiones a partir del debate HevillaEscamilla. Biblio 3W, Revista Bibliográfica de Geografía y Ciencias Sociales, Universidad de Barcelona, España. 


\section{À PARAîTRE}

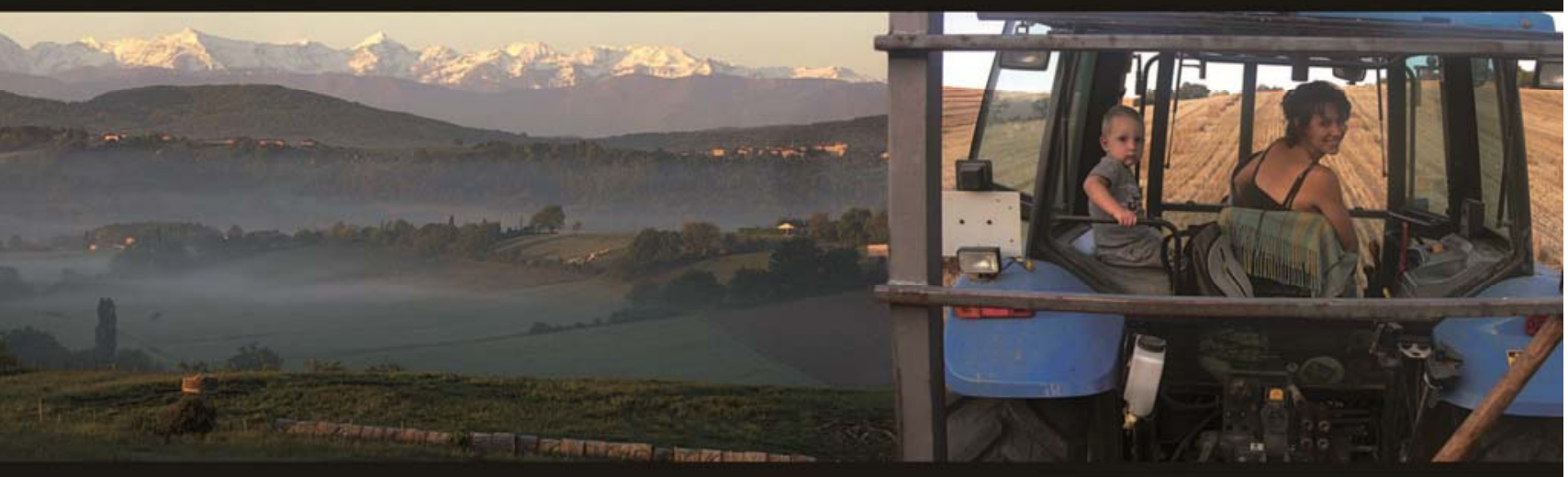

\section{PROfil}

L'agriculture en famille: travailler, réinventer, transmettre

Coordonné par P. Gasselin, J.-P. Choisis, S. Petit, F. Purseigle et S. Zasser

edpsciences 


\section{Présentation}

L'agriculture est-elle encore une affaire de famille ? Cet ouvrage analyse les transformations du caractère familial de l'agriculture en France, mises en perspective avec quelques éclairages de situations dans les pays du Sud.

L'ouvrage rassemble des travaux de recherche récents et des témoignages de chercheurs issus du département Sciences pour l'action et le développement (Sad) de l'Inra et de ses partenaires scientifiques et professionnels. Les auteurs y interrogent l'absence de consensus politique sur ce que doit être l'exploitation agricole, contrairement aux années 1960, exploitation qui est de moins en moins familiale, tout en le restant. La finalité de l'ouvrage est aussi de discuter la coexistence de modèles familiaux, qu'il convient de qualifier et de comprendre, à l'échelle territoriale et dans leur diversité. La famille reste-t-elle un lieu de production et de transmission des connaissances, un support d'identité, un espace social d'innovation pertinent pour envisager la transition agroécologique ? Le caractère familial de l'exploitation, autrefois vecteur d'une cohésion sociale localisée, reste-t-il déterminant dans les nouvelles formes d'action collective entre agriculteurs ? Les problématiques abordées traitent également des nouveaux enjeux de l'agriculture, des transformations des modes d'organisation et d'action collective et des processus d'innovation technique et organisationnelle autour de l'exploitation agricole familiale. L'ouvrage combine des études empiriques localisées, des approches comparatives, des mises en perspective larges et des travaux plus théoriques et conceptuels. Les travaux de recherche, souvent interdisciplinaires, mobilisent l'agronomie, la zootechnie, la sociologie, l'économie, la géographie, les sciences de gestion, l'histoire et l'anthropologie.

La première partie de l'ouvrage rend compte des recompositions de l'exploitation agricole familiale et de ses enjeux. Elle propose des cadres d'analyse élargis pour les appréhender. Les transformations des formes familiales d'agriculture sont abordées dans une deuxième partie par l'étude des mutations qui s'opèrent dans le travail en agriculture. Ces mutations amènent à questionner le sens du travail et les interfaces avec le vivre en famille et les activités non-agricoles, au-delà des seules problématiques d'organisation et de performances technicoéconomiques. La troisième partie de l'ouvrage montre que les cadres structurant l'activité agricole dépassent le champ de la famille et se renouvellent, en particulier dans ses formes d'insertion territoriale et par ses modalités $d^{\prime}$ action collective de proximité. Finalement, les transformations du caractère familial, saisies dans le temps long des trajectoires d'exploitations, sont aussi observées dans les formes et stratégies de pérennisation et de transmission, qu'il s'agisse de transmettre un statut, une activité, une entreprise, un patrimoine, un outil de production ou encore des savoir-faire.

L'ouvrage fait une large place à des travaux de jeunes chercheurs et à des thèses récemment soutenues. II est une contribution du département Sad de l'Inra à l'Année internationale de l'Agriculture familiale 2014. D'abord destiné aux chercheurs et enseignant-chercheurs, il s'adresse également aux organisations professionnelles (instituts techniques, réseaux associatifs, syndicats, chambres d'agriculture, etc.).

Coordination scientifique : Gasselin P., Choisis J.-P., Petit S., Purseigle F. Coordination éditoriale : Zasser $S$.

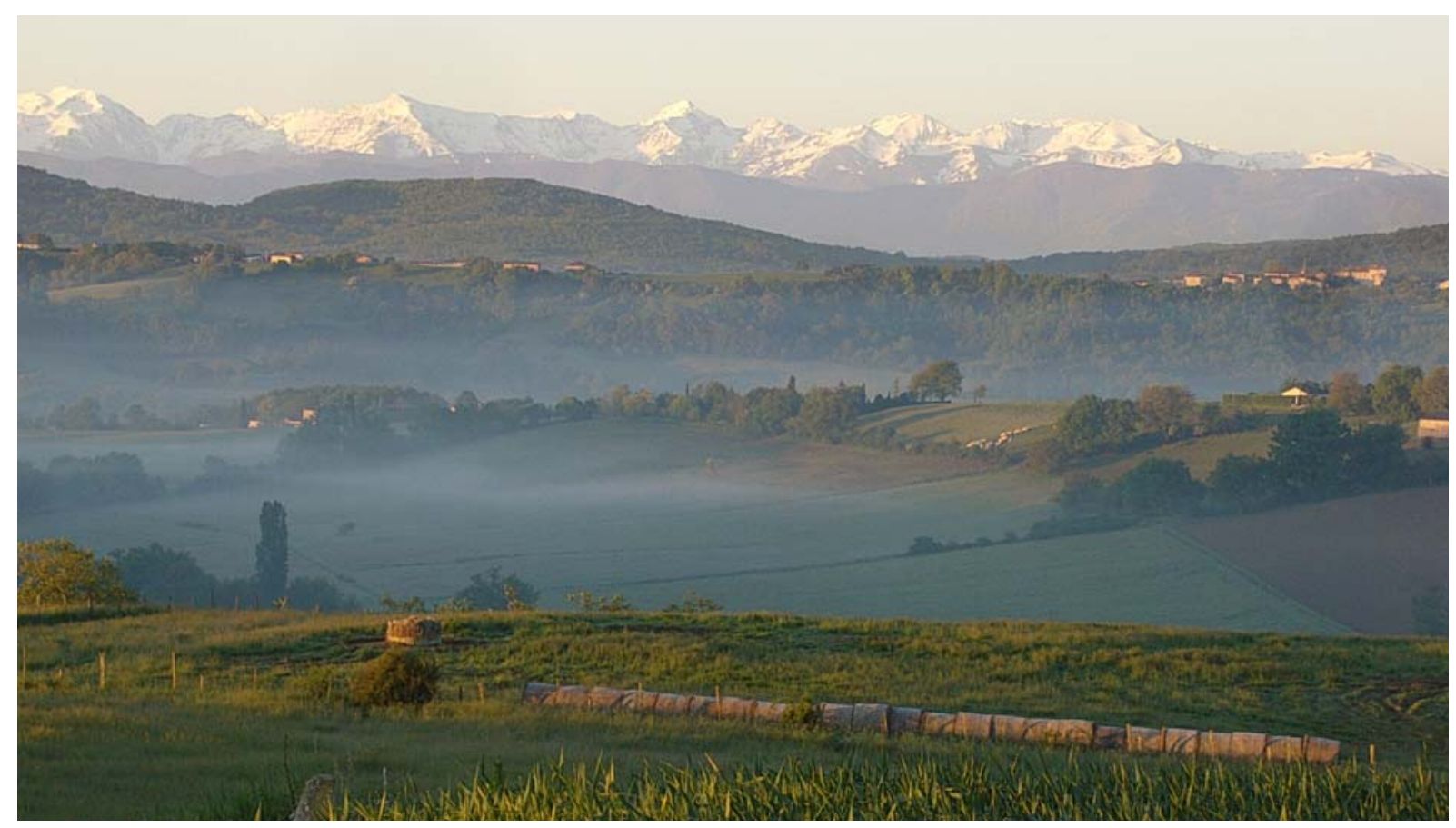

(c) Gérard Balent 


\section{Plan de l'ouvrage}

\section{Préface}

Benoît Dedieu, directeur du département Sciences pour l'action et le développement (Inra)

\section{Introduction}

Par les coordinateurs scientifiques

\section{De l'exploitation agricole à l'agriculture en famille}

1. L'exploitation agricole, entre famille et entreprise, un débat toujours actuel

J. Pluvinage

2. Permanence de l'exploitation agricole familiale, une approche gestionnaire M. Gafsi

3. Unité de l'agriculture et diversité des exploitations agricoles. Des représentations en évolution P. Mundler

4. Théorie du comportement adaptatif et agriculture familiale

E. Chia, M. Petit, J. Brossier

5. Le système d'activité. Retour sur un concept pour étudier l'agriculture en famille

P. Gasselin, M. Vaillant, B. Bathfield

\section{Vivre et travailler en agriculture}

6. Faire face à des questions de travail : les leviers mobilisés par des éleveurs laitiers N. Hostiou, S. Chauvat, S. Cournut

7. Entre maintien et transformation du caractère familial des exploitations agricoles. Le cas des exploitations laitières des Alpes du Nord M. Terrier, S. Madelrieux, B. Dedieu

8. L'évolution de la place des femmes en agriculture au prisme des rapports familiaux de production S. Dahache

9. Famille et élevage : sens et organisation du travail

C. Fiorelli, J. Porcher, B. Dedieu

\section{Réinventer l'agriculture dans le territoire}

10. Coopération Agricole de Production : quand l'activité agricole se distribue entre exploitation et action collective de proximité

V. Lucas, P. Gasselin, F. Thomas, P.-F. Vaquié

11. Transformation de l'unité de production agricole, d'une exploitation sectorielle à une exploitation territoriale ?

P. Vandenbroucke, J. Pluvinage

12. Les agricultures paysannes extrapampéennes argentines. Au-delà des résistances: des modalités innovantes d'articulation agriculture-territoire

R. Cittadini, P. Carricart, R. Bustos Cara, H. Hernán, S. Amalia, S. Patricio, C. Albaladejo

13. Recompositions des liens entre familles et élevages dans des territoires d'élevage herbivores du Nord et du Sud

S. Madelrieux, H. Rapey, C. Corniaux, M.-O. Nozières, J.-P. Choisis, M. Gedouin, J.-P. Dubeuf,

S. Cournut 


\section{Maintenir et transmettre}

14. Quelles stratégies pour un maintien de la polyculture-élevage ? Une étude des trajectoires passées d'exploitations dans les coteaux de Gascogne

J. Ryschawy, N. Choisis, J.-P.Choisis, A. Joannon, A. Gibon

15. Être ou ne pas être agriculteur. Capital scolaire et devenir socioprofessionnel des fils d'agriculteurs C. Giraud, J. Rémy

16. Délégation d'activités et sous-traitance : au service de la transmission de l'exploitation ou d'un patrimoine ?

G. Anzalone, F. Purseigle

17. Transmissions de l'exploitation dans une zone de moyenne montagne pyrénéenne : quelles ruptures, quelles continuités ?

C. Manoli, J.-P.Choisis, S. Ladet, A. Gibon

\section{Conclusion}

Par les coordinateurs scientifiques

\section{Les coordinateurs scientifiques}

Pierre Gasselin est agronome et géographe au département Sciences pour l'action et le développement de l'Inra. Il anime à l'UMR Innovation de Montpellier une équipe sur la coexistence et la confrontation des formes sociales et techniques d'agriculture dans les territoires. Ses travaux en France et en Amérique latine portent sur les transformations de l'activité agricole, son insertion territoriale, les conditions de son accompagnement et les innovations associées.

Jean-Philippe Choisis est zootechnicien système au département Sciences pour l'action et le développement de I'Inra. II aborde des problématiques de développement et de transformation de l'élevage dans les milieux à fortes contraintes à travers l'étude du fonctionnement et de la dynamique des exploitations. Actuellement affecté à l'UMR Dynafor de Toulouse, il a également travaillé en Corse ainsi qu'au Mexique et à la Réunion, sur des terrains de recherche communs avec le Cirad.

Sandrine Petit est géographe au département Sciences pour l'action et le développement de l'Inra, affectée à I'UMR CESAER à Dijon. Ses recherches portent sur les relations entre les agriculteurs et l'environnement. Elles abordent les représentations, les pratiques et les savoirs des agriculteurs liés à l'environnement.

François Purseigle est professeur de sociologie rurale à l'Institut national polytechnique de Toulouse (Ensat), membre de I'UMR Agir et chercheur associé au centre de recherches politiques de Sciences-po Paris. Après avoir conduit des recherches sur le comportement syndical et politique des agriculteurs français, ses travaux portent aujourd'hui sur les nouvelles formes d'organisations économiques et sociales associées aux agricultures de « firme ».

Retrouvez ce livre sur www.edp-open.org (rubrique Books)

Ouvrage en Open Access

Publication : Octobre 2014

Collection PROfil

ISBN : 978-2-7598-1192-2

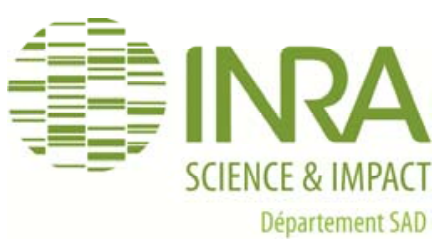

edpsciences

www.edpsciences.org 